\title{
Characterization of Fear Memory Reconsolidation
}

\author{
Sevil Duvarci ${ }^{1}$ and Karim Nader ${ }^{2}$ \\ ${ }^{1}$ W. M. Keck Foundation Laboratory of Neurobiology, Center for Neural Science, New York University, New York, New York 10003, and ${ }^{2}$ Department of \\ Psychology, McGill University, Montreal, Quebec, Canada H3A 1B1
}

\begin{abstract}
Reactivation of consolidated memories returns them to a protein synthesis-dependent state. One interpretation of these findings is that the memory reconsolidates after use. Two alternative interpretations are that protein synthesis inhibition facilitates extinction and that postreactivation protein synthesis inhibition leads to an inability to retrieve the consolidated memory. First, using two different approaches, we report that reconsolidation cannot be reduced down to facilitated extinction. We show that the reconsolidation deficit does not show renewal after a contextual shift, whereas an extinguished auditory fear memory does under the same conditions and the deficit occurs regardless of whether the memory is reactivated with an extinction [conditioned stimulus (CS) alone] or a reinforced trial (CS- unconditioned stimulus). To address the issue of whether postreactivation anisomycin leads to an inability to retrieve the consolidated memory, we used two traditional assays for retrieval deficits. First, we demonstrate that the amnesia induced by blockade of reconsolidation does not show any spontaneous recovery. Second, we show that application of reminder shock does not result in the reinstatement of the memory. These findings support the idea that reactivation of consolidated memories initiates a second timedependent memory formation process.
\end{abstract}

Key words: amygdala; consolidation; reconsolidation; extinction; fear conditioning; plasticity

\section{Introduction}

There are multiple sources of data, including work done with humans (Walker et al., 2003), suggesting that amnesia for the original learning can occur when amnesic treatments are performed immediately after a consolidated, stable long-term memory (LTM) is reactivated (Sara, 2000; Nader, 2003). For example, reactivation of a consolidated fear memory returns it to a labile state, during which it is once again sensitive to protein synthesis inhibition immediately after reactivation but not $6 \mathrm{hr}$ later. These findings suggested that reactivation of consolidated auditory fear memory initiates a second time-dependent memory process (reconsolidation) that again requires protein synthesis to restabilize the original memory (Nader et al., 2000b; Nader, 2003).

Two theoretical challenges to this claim have been posed recently. First, it is argued that protein synthesis inhibition may have caused enhanced consolidation of the extinction memory (Myers and Davis, 2002; Fischer et al., 2004). Typically, the reactivation session is an extinction trial in which the conditioned stimulus (CS) is presented alone. Given that extinction involves new learning (Pavlov, 1927), it is counterintuitive that blocking protein synthesis would facilitate the consolidation of an extinction memory. However, this possibility has not been experimentally ruled out.

Received Feb. 16, 2004; revised Aug. 29, 2004; accepted Sept. 2, 2004.

This work was supported by grants from the Natural Sciences and Engineering Research Council (Canada), The ELB Foundation, International Human Frontiers of Science, and the Volkswagen Foundation to K.N. The Sloan Foundation and the Canadian Institutes of Health Research are acknowledged for their support of K.N. We acknowledge J. E. LeDoux for supporting S.D. during these experiments and comments on previous drafts of this manuscript.

Correspondence should be addressed to Dr. Karim Nader, Department of Psychology, McGill University, 1205 Docteur Penfield Avenue, Montreal, Quebec, Canada H3A 1B1. E-mail: karim.nader@mcgill.ca.

D0I:10.1523/JNEUROSCI.2971-04.2004

Copyright $\odot 2004$ Society for Neuroscience $\quad$ 0270-6474/04/249269-07\$15.00/0
We tested this hypothesis in two ways. First, extinction of fear conditioning is context dependent, such that if the conditioned response is extinguished in one context and the animals are tested in a different context, responding to the CS returns (renews) (Bouton and Bolles, 1979). If the memory deficit induced by protein synthesis inhibition after nonreinforced memory reactivation was attributable to enhanced extinction, then shifting animals to a neutral context should cause their responding to renew. The second approach was to reactivate the consolidated memory with a second reinforced trial and therefore eliminate any extinction during the reactivation session. Thus, if anisomycin is facilitating extinction rather than blocking reconsolidation, giving a tone-shock [CS-unconditioned stimulus (US)] pairing should prevent the memory deficit.

The second theoretical challenge to reconsolidation is the suggestion that anisomycin induces a retrieval failure (the memory is present but cannot be accessed) as opposed to a failure to re-store the information (Cahill et al., 2001; Lattal and Abel, 2004). We performed two traditional assays to test whether the anisomycininduced behavioral deficit after memory reactivation was the result of a deficit in retrieval. First, we tested whether the auditory fear reconsolidation deficit induced by intra-lateral amygdala (LA) administration of anisomycin was long lasting or not. True amnesia, presumably, would not demonstrate any spontaneous recovery. In addition to this, it was previously shown that amnesia induced by electroconvulsive shock after retrieval of a memory could be restored by administration of a reminder shock (Miller and Kraus, 1977). More recent work has also suggested that the memory deficit induced by protein synthesis inhibition after retrieval of the memory can be overcome when amnesic animals are reexposed to the training shock (US) as a reminder cue (Vianna et al., 2001). Therefore, we used reminder shock to 
test whether the animals would recall their memory by the presentation of this reminder cue.

\section{Materials and Methods}

Subjects. Subjects were adult male Sprague-Dawley rats obtained from Hilltop Laboratories (Scottsdale, PA). Rats were housed individually in plastic Nalgene cages and maintained on a $12 \mathrm{hr}$ light/dark cycle. Food and water were provided ad libitum throughout the experiment.

Surgery and histology. Under Nembutal anesthesia (45 mg/kg), rats were implanted bilaterally with 22 gauge stainless steel guide cannulas into the LA. Coordinates, taken from Paxinos and Watson (1986), were $3.0 \mathrm{~mm}$ posterior to bregma, $5.3 \mathrm{~mm}$ lateral to the midline, and $8.0 \mathrm{~mm}$ ventral to the skull surface. Rats were given at least $7 \mathrm{~d}$ to recover before experimental procedures.

At the end of the experiment, using standard histological methodologies, animals were perfused, and their brains were sectioned at $50 \mu \mathrm{m}$ thickness. The sections were stained using cresyl violet and examined with light microscopy for cannula penetration into the lateral and basal nuclei. All procedures were in accordance with the NIH Guide and were approved by the New York University Animal Care and Use Committee.

Intra-lateral and basal amygdala infusions. Using a 28 gauge injector, drugs were infused slowly via an infusion pump into the lateral and basal amygdala (LBA) at a rate of $0.25 \mu \mathrm{l} / \mathrm{min}$. After drug infusion, injectors were left in place for one additional minute to allow diffusion of the drug away from the cannula tip. Anisomycin (catalog \#A9789; Sigma, St. Louis, $\mathrm{MO}$ ) was dissolved in equimolar $\mathrm{HCl}$, diluted with artificial CSF (ACSF), and adjusted to $\mathrm{pH} 7.4$ with $\mathrm{NaOH}$. Although the lateral nucleus was the main target, the $0.5 \mu \mathrm{l}$ infusions probably affected the adjacent basal nucleus. We, therefore, refer to the affected area as the LBA. For all anisomycin experiments, the lowest dose of anisomycin that affects both consolidation and reconsolidation $(62.5 \mu \mathrm{g} / 0.5 \mu \mathrm{l} /$ side) was used (Nader et al., 2000b; Schafe and LeDoux, 2000).

Apparatus. Conditioning and tone testing were conducted in different chambers. For conditioning, rats were placed in a Plexiglas rodent conditioning chamber (context A) with a metal grid floor (model E10-10; Coulbourn Instruments, Lehigh Valley, PA) that was enclosed within a sound attenuating chamber (model E10-20). The chamber was dimly illuminated by a single house light. For tone testing, rats were placed in a different Plexiglas chamber (ENV-001; MedAssociates, Georgia, VT). In the tone-testing chamber, two distinctly different contexts were used. Context B was brightly lit with three house lights on and contained a flat black Formica floor that had been washed with peppermint soap. Context $\mathrm{C}$ was lit with three flashing house lights and contained bedding under a flat black Formica floor and the sound of a fan. A micro-video camera was mounted at the top of the chamber so that rats could be videotaped during testing.

General behavioral procedures. In all of the experiments, rats were habituated to conditioning and testing contexts for 10 min each on day 0 in a counterbalanced manner. The training trials consisted of a single auditory conditioning trial in which the auditory CS was a $30 \mathrm{sec}$ presentation of a $5 \mathrm{kHz}, 80 \mathrm{~dB}$ tone CS that coterminated with a $1.5 \mathrm{~mA}, 1 \mathrm{sec}$ footshock US. Memory reactivation (except in experiment 2 ) was performed by administering a single $30 \mathrm{sec}$ CS. For all tests, rats received a $30 \mathrm{sec}$ presentation of three conditioned stimuli. Seconds of freezing during the CS presentations were scored for each rat, and the scores were shown as the percentage of the total duration of CS presentation.

Experiment 1. Rats were habituated to the conditioning (context A) and the two testing (contexts B and C) contexts for 10 min each on day 0 in a counterbalanced order. On day 1, they were placed into context A and were given a single conditioning trial. Rats were then returned to their home cages. On day 2, after CS reactivation in context B, rats received either anisomycin [Same, $n=8$; different (Diff), $n=11$ ] or ACSF (Same, $n=7$; Diff, $n=8$ ) infusions. Twenty-four hours later, rats were returned to context $\mathrm{B}$ and received three CS presentations [postreactivation LTM (PR-LTM) test]. The ACSF rats continued to receive nonreinforced CS presentations (10-13 conditioned stimuli) until their score was comparable with anisomycin rats. To equate for the duration ACSF animals spend in the context until their freezing behavior extinguished, the anisomycin-treated animals were left in the boxes for the same dura- tion without receiving any CS presentations (30-40 min). The next day, anisomycin/Same and ACSF/Same animals were returned to context B, whereas anisomycin/Diff and ACSF/Diff animals were placed in a third context (context C) to receive their final test. Contexts $\mathrm{B}$ and $\mathrm{C}$ were counterbalanced such that half the animals were extinguished in $\mathrm{C}$ and the other half were extinguished in $\mathrm{B}$.

Experiment 2. After habituation to contexts A and B, rats received one auditory fear conditioning per day for $2 \mathrm{~d}$ in context A. All rats received an infusion of either ACSF or anisomycin after each trial. Thus, the experiment was composed of four groups [A/V $(n=7) ; \mathrm{A} / \mathrm{A}(n=5)$; V/A $(n=8) ; \mathrm{V} / \mathrm{V}(n=9)]$, in which the first and second letters refer to whether animals received anisomycin (A) or ACSF vehicle (V) after the first and second training trial, respectively. Twenty-four hours later, animals were placed in context B and received three CS presentations (final test). Day 2 will act as the equivalent of retraining for the $\mathrm{A} / \mathrm{V}$ group.

Consolidation and reconsolidation make differential predictions concerning the behavior of group V/A. The consolidation theory predicts that the anisomycin infusions after the second training day should only block the consolidation of the new information acquired during that trial, while sparing the consolidated memory for trial 1 (McGaugh, 1966). Thus, in the final test, group V/A should freeze at the same level as on day 2. Reconsolidation, in contrast, predicts that when the CS is presented on day 2 , the consolidated memory of trial 1 returns to a labile state and the new information acquired on day 2 should also be in a labile state. Thus, anisomycin treatment should block all memory for the task, causing V/A animals to behave like animals in the A/A group in the final test.

Experiment 3. After habituation to contexts A and B, rats received a single conditioning trial in context $\mathrm{A}$, which was followed, $24 \mathrm{hr}$ later, by reactivation of the memory with a $30 \mathrm{sec} C S$ presentation in context $\mathrm{B}$. Rats were infused with either $62.5 \mu \mathrm{g}$ per $0.5 \mu \mathrm{l}$ per side of anisomycin $(n=7)$ or ACSF $(n=7)$ immediately after CS termination of the reactivation session. This is the effective dose of anisomycin to block both consolidation and reconsolidation (Nader et al., 2000b; Schafe and LeDoux, 2000). A postreactivation short-term memory (PR-STM) test was performed $4 \mathrm{hr}$ later, followed by a PR-LTM test $24 \mathrm{hr}$ after reactivation. Rats were then left in their home cage for $23 \mathrm{~d}$. Twenty-four days after reactivation, they were returned to context $\mathrm{B}$ and given three CS presentations (final test). The next day, anisomycin rats were returned to context $\mathrm{A}$ and were retrained by being given two CS-US presentations, consisting of a $30 \mathrm{sec}$ presentation of a $5 \mathrm{kHz}, 80 \mathrm{~dB}$ tone CS that coterminated with a $1.5 \mathrm{~mA}, 1 \mathrm{sec}$ footshock US. Twenty-four hours later, rats were returned to context B and received three CS presentations for testing their retrained LTM.

Experiment 4. The experimental procedure until the end of the reactivation session, followed by either anisomycin $(n=7)$ or ACSF $(n=7)$ infusions, was identical to experiment 3 . Twenty-four hours after reactivation, the rats received a PR-LTM test consisting of three CS presentations. The next day, the animals were taken to a neutral context and given a reminder footshock $(1.5 \mathrm{~mA}, 1 \mathrm{sec})$ that was identical to that used during training. For the reminder shock administration, we used the identical protocol of Vianna et al. (2001) that was shown to result in the reinstatement of a previously amnesic memory. This protocol is also in accordance with the protocol used by Miller and Kraus (1977) that the reminder shock is given in a neutral context. Twenty-four hours after the reminder shock administration, animals underwent a test for memory reinstatement that consisted of three CS presentations in context $\mathrm{B}$. The next day, rats were retrained in context A with a single CS-US trial consisting of a $30 \mathrm{sec}, 5 \mathrm{kHz}, 80 \mathrm{~dB}$ tone CS that coterminated with a 1.5 $\mathrm{mA}, 1$ sec footshock US. The following day, they were tested for the LTM of the retrained memory by three CS presentations in context B.

\section{Results \\ Does the reconsolidation deficit, when induced by a nonreinforced trial, resemble extinction?}

Extinction is context dependent such that conditioned responding renews when animals are tested in a context that is different from the one in which extinction occurred. If the effects of ani- 


\section{a}

CONTEXTA

CONTEXTB

FINAL TEST

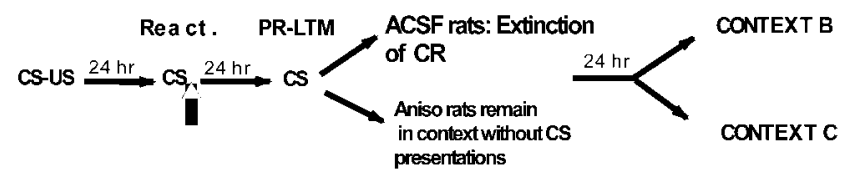

b

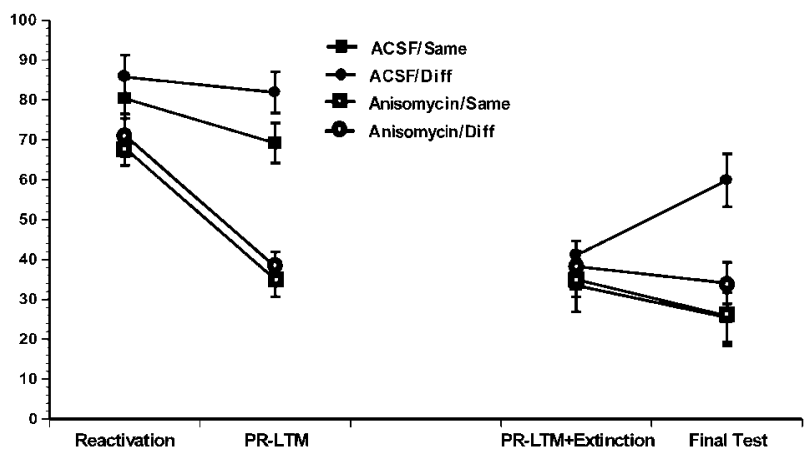

Figure 1. The impairment induced by postreactivation anisomycin infusions is not context specific. $a$, The behavioral procedure used for experiment 1. $b$, Anisomycin groups were impaired in the PR-LTM test. The first word in the group name refers to whether animals received anisomycin or ACSF infusions. The second word refers to whether they will be tested in the same (Same) or different (Diff) context.

somycin infused after nonreinforced reactivation are attributable to accelerated extinction, then shifting the animals to another context should cause their responding to increase or "renew" (Bouton and Bolles, 1979). However, if the effects of anisomycin are attributable to blockade of "restorage" of the memory trace, there should be no effect of a shift to a novel context because there is no effective memory present. To evaluate this possibility, we induced a reconsolidation deficit by infusing anisomycin after CS reactivation. Vehicle-treated animals continued to receive nonreinforced conditioned stimuli until their freezing levels were comparable with the anisomycin-treated rats (Fig. 1a). To maximize the anisomycin-treated rats' ability to establish a strong contextual representation, which would facilitate renewal, they were left in the reactivation context (context B) for the same amount of time it required for the control animals to extinguish. The following day, the anisomycin and ACSF groups were divided into two subgroups. One of the subgroups from each treatment condition was tested the next day in the same context as they had been previously tested and extinguished (ACSF/Same, anisomycin/Same), and the other subgroup was tested in a different neutral context (ACSF/Diff, anisomycin/Diff).

All animals demonstrated comparable reactivation scores and groups that received anisomycin postreactivation demonstrated impaired PR-LTM (Fig. 1a,b). A repeated-measures ANOVA comparing drug (anisomycin vs ACSF) with trial (reactivation vs PR-LTM) and contextual manipulation (same vs different), revealed no significant three-way interaction of these groups $(F<$ 1 ). This is not surprising because the animals had not yet received any contextual manipulation. There was a significant two-way interaction between drug and trial (see Fig. $3 b$, left $)\left(F_{(1,30)}=24\right.$; $p<0.05)$ demonstrating that the anisomycin-treated animals were significantly impaired compared with both their own reactivation scores and to the PR-LTM scores of the ACSF groups $(p<0.05)$. In addition, the two anisomycin groups demonstrated comparable scores with each other as did the ACSF groups $(p>0.05)$.

The ACSF/Diff, but not anisomycin/Diff, animals showed renewed responding when tested in a different context (Fig. 1b). A three-way ANOVA comparing the drug group (anisomycin vs ACSF), trial (the last three tone presentations of the vehicle groups and the PR-LTM scores of the anisomycin groups vs freezing on the final test), and contextual manipulations (same vs different) did not reveal a significant interaction (Fig. 1b, right) $\left(F_{(1,30)}=2.1 ; p=0.15\right)$. Because there is a visual trend for an interaction that our analysis may not have had the power to detect, we performed post hoc comparisons on the analyses. As suspected, the ACSF/Diff group demonstrated significantly more freezing on the final test compared with all other groups either for day 3 or final tests $(p<0.05)$. To further reexamine the validity of this effect, we used separate two-way ANOVAs on the scores of animals that were shifted to a different context and a separate one for animals that were not. An ANOVA comparing the drug group and trial for the Diff animals revealed a significant interaction $\left(F_{(1,17)}=6 ; p<0.05\right)$. A similar analysis on the scores of animals that received their final test in the same context revealed no interaction between drug and trial $(F<1)$. Thus, consistent with a large literature on extinction, our ACSF animals showed renewal if they were shifted to a neutral context. The anisomycin-treated animals, however, did not demonstrate renewal, demonstrating that the anisomycin deficit does not follow the classical behavioral pattern characteristic of extinction.

All rats included in the behavioral analyses had bilaterally placed injectors in the LBA (Fig. 2). All other animals were excluded from the analyses (cannula placements in the other experiments reported below are similar to the ones presented here and are not shown).

Recently, similar injections of anisomycin have been shown to reduce protein synthesis inhibition over a large area that expands beyond the amygdala (Maren et al., 2003). We are confident that our effects are attributable to an action in the LBA because the results from lesion, pharmacological, and electrophysiological studies have shown that the LA and not surrounding tissue is critical for the acquisition of fear memories (LeDoux, 2000; Maren, 2001). The findings that anisomycin injections into the LBA, but not dorsal to it, block consolidation of fear memories demonstrates that the LBA is critical for consolidation (Schafe and LeDoux, 2000). Finally, animals that are disqualified from the analyses based on misplaced cannula do not show a significant impairment, although the cannulas were close to the LBA.

\section{Reactivation by a reinforced trial}

Two conditioning sessions were performed on consecutive days in which animals received one tone-shock pairing and then received the final test the following day. According to a Latinsquare design, after each conditioning session on days 1 and 2, each rat received either an infusion of vehicle (V) or anisomycin (A) (Fig. 3a). This produced four 4 groups (V/V, A/V, V/A, and $\mathrm{A} / \mathrm{A}$ ) in which the first and second letters of the group names refer to the treatments after the first and second pairings, respectively.

As can be seen, animals in group V/A clearly show a marked decrease in freezing behavior between day 2 and the final test (Fig. $3 b)$. A $3 \times 3$ ANOVA comparing day 1 infusions (anisomycin or vehicle) with day 2 infusions (anisomycin or vehicle) and trial (day 1, day 2, and final test) did not demonstrate a significant interaction $(F<1)$. There was a significant two-way interaction between the day 1 treatment and trial $\left(F_{(2,50)}=43 ; p<0.05\right)$ 
a
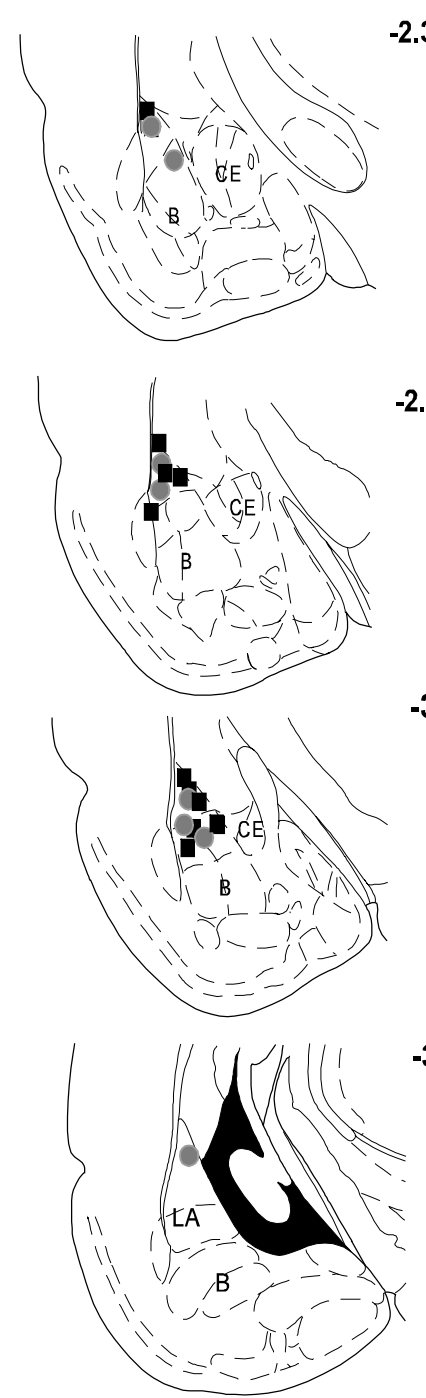

b
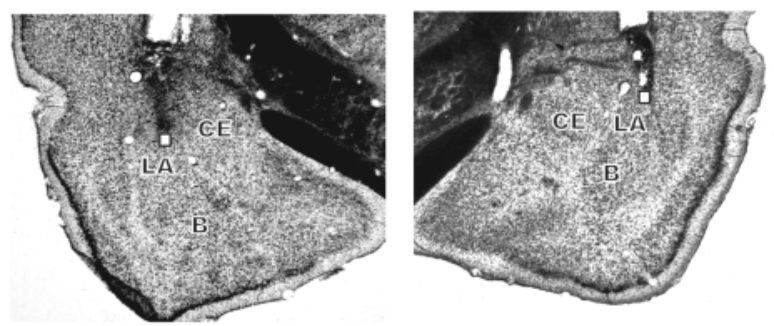

Figure 2. a, Schematic representation of the amygdala at four different rostrocaudal planes. The numbers represent the posterior coordinate from bregma. Injector placements in the LBA are represented by the filled symbols; black filled squares represent Aniso/Different group placements, gray filled circles represent anisomycin/Same group placements. The placements for the other groups in this experiment and subsequent experiments all demonstrate similar distributions and therefore are not shown. $b$, Photomicrographs of representative cannula placements (white square) in the BLA. The left photomicrograph is taken from an animal that received vehicle, and the right is from an animal that received anisomycin. $L A$, Lateral nucleus; $\mathrm{B}$, basal nucleus; $\mathrm{CE}$, central nucleus.

showing that anisomycin infusions caused memory impairments in subsequent days. Importantly, there was a similar significant interaction between day 2 treatment and trial $\left(F_{(2,50)}=57 ; p<\right.$ $0.05)$ demonstrating that anisomycin infusions on day 2 had sig- a

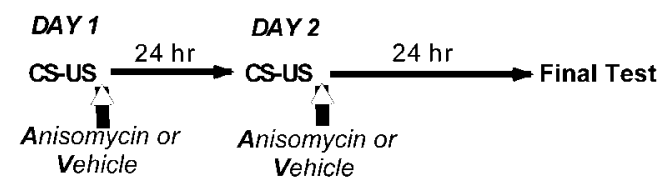

b

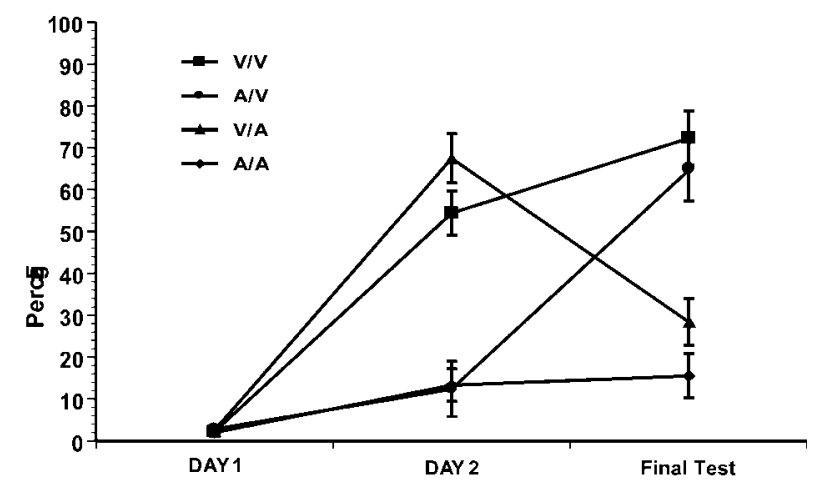

Figure 3. Consolidated memories that are reactivated by a reinforced trial are sensitive to protein synthesis inhibition. $a$, The behavioral procedure used for experiment 2 . Vertical openheaded arrows represent intra-LBA infusions in all figures. $b$, The first and second letter of the group name refer to the treatment received ( $A$, anisomycin; $V$, vehicle ACSF) after the first and second training trial, respectively. The score of animals in group V/A sharply declined between day 2 and the final test. This is consistent with the predictions of reconsolidation but not consolidation.

nificant memory impairments independent of the freezing scores in day 1. Newman-Keuls post hoc analysis revealed that all groups demonstrated comparable behavior on day $1(p>0.05)$. On day 2 , the $\mathrm{V} / \mathrm{V}$ and $\mathrm{V} / \mathrm{A}$ groups demonstrated comparable freezing levels that were both significantly higher than either the A/A or $\mathrm{A} / \mathrm{V}$ groups $(p<0.05)$, the latter two being comparable with each other $(p>0.05)$. On the final test, both the V/V and $\mathrm{A} / \mathrm{V}$ groups demonstrated a significant increase in freezing from the previous day, whereas the $\mathrm{A} / \mathrm{A}$ group demonstrated comparable freezing to day 2. Critically, the V/A group demonstrated a dramatic decrease from day 2 freezing levels $(p<0.05)$. This demonstrates that auditory fear memories in the amygdala return to a labile state when reactivated with a reinforced trial.

In addition, it should be noted that consolidation and reconsolidation make differential predictions concerning the behavior of group V/A. The consolidation theory predicts that the anisomycin infusions after the second training day should only block the consolidation of the new information acquired during the second trial while sparing the consolidated memory for trial 1 (McGaugh, 1966). Thus, in the final test, group V/A should freeze at the same level as on day 2 because only the new learning on day 2 should be in a labile state. Reconsolidation, in contrast, predicts that when the CS is presented on day 2, the consolidated memory of trial 1 returns to a labile state and the new information acquired on day 2 should also be in a labile state. Thus, anisomycin treatment should block all memory for the task causing V/A animals to behave like animals in the A/A group in the final test. Therefore the finding that group V/A decreased its responding toward group A/A cannot be described by the consolidation theory and is consistent with reconsolidation.

\section{Is the reconsolidation deficit long lasting?}

In our previous study (Nader et al., 2000b), rats received a PRSTM and PR-LTM tests at 4 and $24 \mathrm{hr}$ after reactivation, respectively. We used the same protocol, with the exception that an 
a

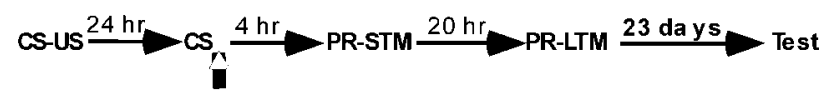

b

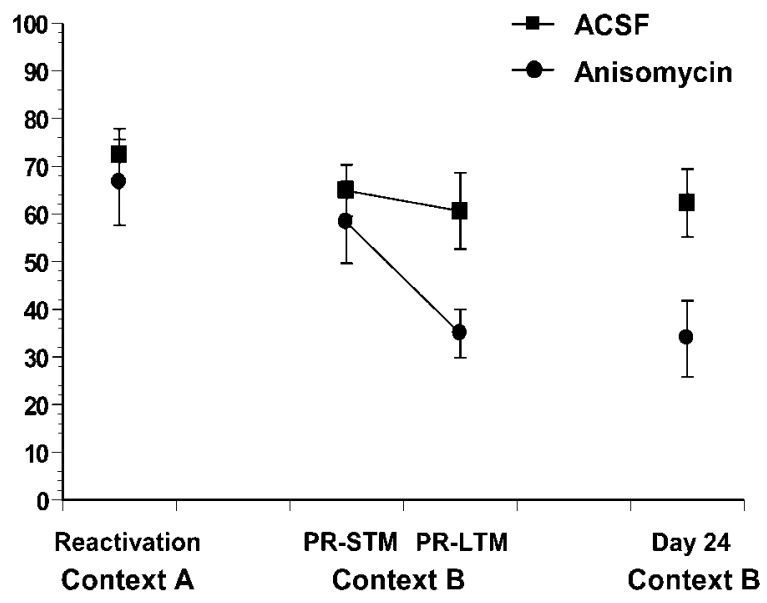

Figure 4. The amnesia resultant from anisomycin infusions does not show spontaneous recovery over 24 d. $a$, Schematic of behavioral procedure used. $b$, Anisomycin infusions impaired PR-LTM but had no effect on PR-STM. Twenty-four days after reactivation, anisomycin rats were still impaired.

additional test was performed $24 \mathrm{~d}$ after reactivation to test spontaneous recovery. The freezing scores during reactivation for the anisomycin and vehicle animals did not differ significantly $\left(t_{(12)}=0.5 ; p>0.05\right)$ (Fig. $\left.4 b\right)$. An ANOVA comparing the drug group (ACSF and anisomycin) across trials (PR-STM, PR-LTM, and final tests) demonstrated a significant interaction $\left(F_{(2,24)}=\right.$ 5.6; $p<0.05)$. Newman-Keuls post hoc analyses revealed that although the two groups demonstrated comparable PR-STM $(p>0.05)$, anisomycin-infused rats were significantly different from control rats in the PR-LTM $(p<0.05)$. This behavioral deficit did not demonstrate any spontaneous recovery over $23 \mathrm{~d}$. Post hoc analyses demonstrated that the anisomycin- and vehicletreated animals both had comparable behavior with their respective PR-LTM scores 23 d later $(p>0.05)$.

It is possible that both the behavioral impairment in PR-LTM and the absence of spontaneous recovery in the anisomycin group were attributable to damage to the amygdala. To evaluate this possibility, anisomycin-treated rats were retrained. The freezing exhibited by the anisomycin rats during their LTM test was comparable with their freezing during the reactivation test $\left(t_{(7)}=1 ; p>0.05\right)$. Thus, the impairments in the PR-LTM and the absence of spontaneous recovery are not attributable to permanent damage to the amygdala preventing animals from freezing, and suggest that the behavioral deficit that results from blockade of reconsolidation is long lasting.

\section{Does a reminder shock result in recovery of the memory?}

Animals were trained according to our previous reconsolidation paradigm and then tested for whether the memory would recover from amnesia by the application of reminder shock as a reminder cue for the training memory. For reminder shock administration, the protocol of Vianna et al. (2001), who demonstrated that postreactivation blockade of protein synthesis induced amnesia recovered after application of a reminder shock, was used. An ANOVA on the reactivation, PR-LTM, and reinstatement ses-

\section{a}
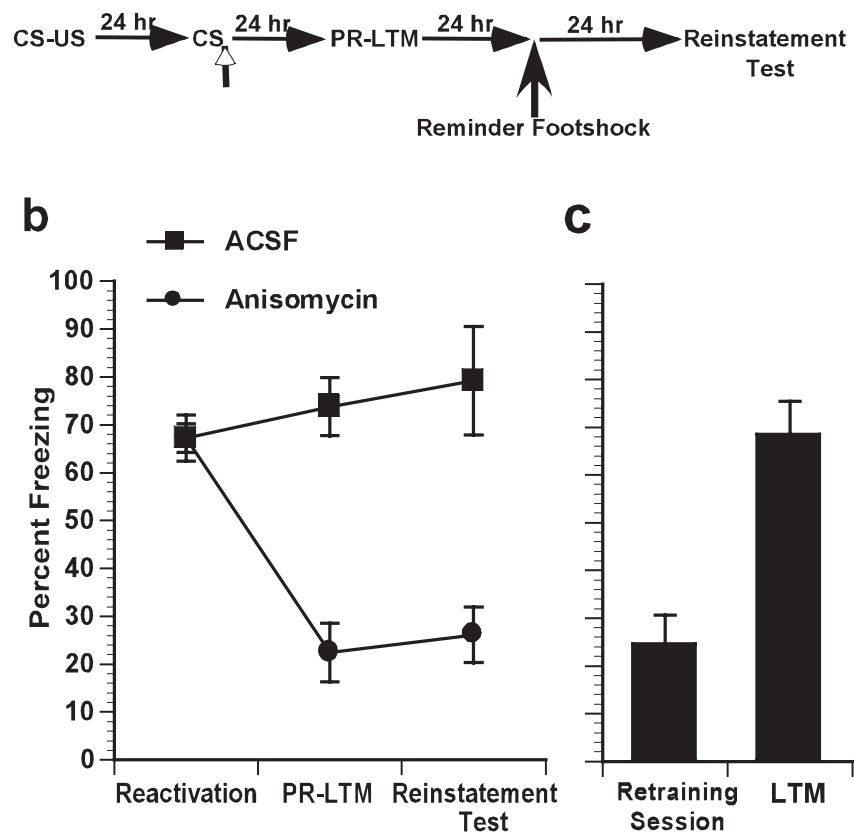

Figure 5. The amnesia resultant from anisomycin infusions does not show reinstatement. $a$, Schematic of behavioral procedure used. Vertical open-headed arrows represent intra-LBA infusions in all figures. $b$, Anisomycin infusions impaired PR-LTM. Animals did not recover from this deficit with a noncontingent footshock. $c$, After retraining, the freezing scores of the anisomycin rats were comparable with their reactivation score, demonstrating that they have a functional amygdala.

sions demonstrated a significant interaction with drug treatment (Fig. $5 a, b)\left(F_{(2,20)}=32 ; p<0.05\right)$. Post hoc comparisons showed that reactivation scores were the same for both groups $(p>$ $0.05)$, whereas the anisomycin-treated animals demonstrated less freezing than controls on both the PR-LTM and reinstatement tests $(p<0.05)$. Furthermore, there was no increase in the freezing scores of anisomycin-treated animals after the reminder shock. To rule out the possibility that the lack of recovery was attributable to anisomycin-induced damage to the amygdala, animals were again retrained. Anisomycin-treated animals significantly increased their freezing after retraining $\left(t_{(5)}=10 ; p<\right.$ $0.05)$ to levels comparable with control animals on reactivation. This demonstrates that the lack of reinstatement is not attributable to the inability of animals to freeze (Fig. $5 c$ ).

\section{Discussion}

The experiments presented here were aimed at addressing two alternative interpretations of our previous results that have become central issues in the debate concerning reconsolidation. First, it has been proposed that anisomycin-induced deficits are not attributable to a blockade of reconsolidation of the original memory but rather to a facilitation of extinction consolidation (Myers and Davis, 2002; Fischer et al., 2004). We directly tested this possibility using two distinct lines of experimentation. We first asked whether the anisomycin deficit induced by memory reactivation using a nonreinforced CS acted in a similar way as an extinguished fear memory and second whether a comparable deficit would be produced in situations in which the memory was reactivated in the absence of extinction.

Control animals that received extinction trials demonstrated renewal when they were tested in a different context. This replicates the well established contextual control of fear extinction 
(Bouton and Bolles, 1979). Anisomycin-treated rats did not show any renewal when tested in the different context. The lack of renewal was not attributable to differential levels of freezing on the day before the contextual shift because the control animals exhibited freezing levels comparable with anisomycin-treated animals at the end of extinction. Furthermore, the lack of renewal is unlikely to be attributable to amygdala damage because animals that received almost identical treatment in experiments 3 and 4 could be retrained and can show intact PR-STM (Nader et al., 2000b). The lack of renewal is consistent with the view that anisomycin blocked the reconsolidation of the auditory fear memory, and thus testing animals in a different context should have minimal effects on responding levels. Given that the anisomycin-induced behavioral deficit behaves differently from extinction, it is clear that reconsolidation and extinction are not reducible to one another. This conclusion is strengthened by the recent findings that extinction and reconsolidation can be double dissociated from each other, depending on which trace is dominant after reactivation (Eisenberg et al., 2003; Pedreira and Maldonado, 2003).

To test whether reconsolidation deficits could be observed in the absence of extinction, we reactivated consolidated auditory fear memories using reinforced trials. We found that animals in group V/A demonstrated a dramatic drop in behavior after day 2, consistent with the predictions of reconsolidation and violating the predictions of facilitation of extinction. This decrease in behavior by group V/A is unlikely to be attributable to anisomycin damage because animals in the $\mathrm{A} / \mathrm{V}$ group, which also received a single injection of anisomycin, demonstrated strong acquisition between day 2 and the final test. These findings demonstrate that reconsolidation occurs in the amygdala in the absence of extinction and therefore categorically rules out facilitated extinction interpretations of reconsolidation.

The second theoretical issue proposes that the reconsolidation deficit is attributable to a failure to retrieve an existing memory as opposed to failure to restore the original memory. In contrast, amnesia resulting from blockade of consolidation is accepted as a storage impairment of the new memory (Cahill et al., 2001; Lattal and Abel, 2004). In our study, the anisomycin-induced deficit did not show spontaneous recovery over $24 \mathrm{~d}$. This is consistent with the absence of recovery after inhibiting reconsolidation in the hippocampus of contextual representation used in contextual fear conditioning (Debiec et al., 2002).

Using a contextual fear conditioning paradigm in conjunction with systemic anisomycin injections, it has recently been demonstrated that the impairment induced by postreactivation anisomycin recovered over time, whereas the impairment in consolidation did not (Lattal and Abel, 2004). The authors suggest that blockade of reconsolidation leads to a transient retrieval-impairment of an existing memory. There are a number of issues with these interpretations. First, to demonstrate that a behavioral impairment is attributable to consolidation blockade, and not nonspecific effects of a manipulation, intact STM must be demonstrated (Dudai, 2004). Similarly, to conclude that a manipulation is affecting the reconsolidation process specifically, intact PRSTM must be demonstrated (Nader, 2003). This study did not test for either STM or PR-STM, so the impairment induced by the systemic anisomycin may be a nonspecific one in nature. In the absence of this data, there would seem to be no grounds for using the terms "consolidation" or "reconsolidation." The same argument is especially important for studies that use prereactivation infusions (Vianna et al., 2001), because the presence of the drug during reactivation could cause a behavioral impairment that is not related to reconsolidation. Given that Vianna et al. (2001) did not test for PR-STM, then it is not clear that the reported deficit is related to reconsolidation. The majority of studies that have tested for recovery and reported intact PR-STM and impaired PR-LTM have not found recovery from amnesia (Debiec et al., 2002; Bozon et al., 2003; Child et al., 2003; Pedreira and Maldonado, 2003; Suzuki et al., 2004), whereas one group has reported recovery in newborn chicks (Anokhin et al., 2002; Salinska et al., 2004). Thus, at this point, there is little modern evidence for spontaneous recovery from a specific impairment in reconsolidation.

Second, if the systemic anisomycin injections produced specific effects in the study by Lattal and Abel (2004), it is well established that the probability of recovery from consolidation blockade is an inverse function of the behavioral impairment induced by the amnesic treatment (Cherkin, 1972; Gold et al., 1973; Davis and Rosenzweig, 1978). This increased tendency to observe recovery from smaller behavioral impairments can explain the differential recovery in the study by Lattal and Abel (2004). On the first test, postreactivation anisomycin induced an $\sim 35 \%$ deficit, whereas the blockade of consolidation induced a $70 \%$ deficit. Thus, the smaller impairment of reconsolidation should have a higher probability of recovery. In another study on reconsolidation in the hippocampus, no recovery was seen over $28 \mathrm{~d}$, and the performance impairment that resulted from reconsolidation blockade was $80 \%$ (Debiec et al., 2002). The magnitude of this impairment is comparable with the consolidation impairment reported by Lattal and Abel (2004), which did not recover. These differences in baselines of amnesia preclude any conclusions as to the qualitative nature of amnesia induced by reconsolidation challenge. This is because it is unclear whether the observed recovery from the reconsolidation impairment occurred because of the different levels of amnesia between the reconsolidation and consolidation experiments or whether recovery is more likely to result from a reconsolidation impairment, regardless of how profound the amnesia is. Given that it is already established that recovery from consolidation blockade is a function of the magnitude of amnesia (Cherkin, 1972; Gold et al., 1973; Davis and Rosenzweig, 1978), the former interpretation would seem most likely. We should not forget that there are some reports of spontaneous recovery from amnesia induced by consolidation blockade (Kohlenberg and Trabasso, 1968; Quartermain and McEwen, 1970; Serota, 1971; Young and Galluscio, 1971).

In addition to a lack of spontaneous recovery, we found no evidence for reminder shock-induced recovery from amnesia. Using a protocol that induces reinstatement (Vianna et al., 2001), we found that the anisomycin deficit did not recover after a reminder shock. The absence of recovery from amnesia was not attributable to permanent damage to the amygdala in the anisomycin-treated animals because they could be retrained, and we found that postreactivation intra-LA anisomycin of this dose has no effect on overtrained fear memories (S. Wang and $\mathrm{K}$. Nader, personal communication). It should be noted that reinstatement in simple paradigms can be attributable to the reminder shock trial acting as a new learning session, which leads to increased responding as opposed to overcoming a retrieval impairment (Gold et al., 1973). A recent study, however, has moved this debate forward by demonstrating reinstatement in a water maze in a manner that is extremely hard for the new learning interpretation of reinstatement to explain (de Hoz et al., 2004). There is no unique interpretation to our lack of spontaneous recovery or reminder shock-induced recovery. The absence of 
recovery is equally consistent with both retrieval and storage impairment interpretations of amnesia.

Currently, we do not think there are established paradigms to directly test whether amnesia is attributable to a retrieval or storage deficit in simple paradigms (Gold and King, 1974; Miller and Springer, 1974; Nader et al., 2000a). For this reason, we have argued that we are agnostic with regard to the qualitative nature of amnesia after blockade of consolidation and reconsolidation (Debiec et al., 2002; Nader, 2003). Our emphasis, however, is specifically on reconsolidation being the reiteration of another time-dependent memory process that is analogous to new memory formation and not on whether that memory process is a retrieval or storage process (Nader, 2003). We use the traditional storage terminology in our explanation and interpretations because it is the dominant memory model within neurobiology. To understand the nature of amnesia, we must develop new paradigms and approaches such as the one that was used in the water maze (de Hoz et al., 2004).

\section{References}

Anokhin KV, Tiunova AA, Rose SP (2002) Reminder effects-reconsolidation or retrieval deficit? Pharmacological dissection with protein synthesis inhibitors following reminder for a passive-avoidance task in young chicks. Eur J Neurosci 15:1759-1765.

Bouton ME, Bolles RC (1979) Contextual control of the extinction of conditioned fear. Learn Motiv 10:445-466.

Bozon B, Davis S, Laroche S (2003) A requirement for the immediate early gene zif268 in reconsolidation of recognition memory after retrieval. Neuron 40:695-701.

Cahill L, McGaugh JL, Weinberger NM (2001) The neurobiology of learning and memory: some reminders to remember. Trends Neurosci 24:578-581.

Cherkin A (1972) Retrograde amnesia in the chick: resistance to the reminder effect. Physiol Behav 8:949-955.

Child FM, Epstein HT, Kuzirian AM, Alkon DL (2003) Memory reconsolidation in hermissenda. Biol Bull 205:218-219.

Davis HP, Rosenzweig MR (1978) Recovery as a function of the degree of amnesia due to protein synthesis inhibition. Pharmacol Biochem Behav 8:701-710.

Debiec J, LeDoux JE, Nader K (2002) Cellular and systems reconsolidation in the hippocampus. Neuron 36:527-538.

de Hoz L, Martin SJ, Morris RG (2004) Forgetting, reminding, and remembering: the retrieval of lost spatial memory. Public Library Sci 2:1233-1242.

Dudai Y (2004) The neurobiology of consolidations, or, how stable is the engram? Annu Rev Psychol 55:51-86.

Eisenberg M, Kobilo T, Berman DE, Dudai Y (2003) Stability of retrieved memory: inverse correlation with trace dominance. Science 301:1102-1104.

Fischer A, Sananbenesi F, Schrick C, Spiess J, Radulovic J (2004) Distinct roles of hippocampal de novo protein synthesis and actin rearrangement in extinction of contextual fear. J Neurosci 24:1962-1966.

Gold P, King R (1974) Storage failure versus retrieval failure. Psychol Rev 81:465-469.

Gold PE, Haycock JW, Marri J, McGaugh JL (1973) Retrograde amnesia and the "reminder effect": an alternative interpretation. Science 180:1199-1201.

Kohlenberg R, Trabasso TOM (1968) Recovery of a conditioned emotional response after one or two electroconvulsive shocks. J Comp Physiol Psychol 65:270-273.

Lattal KM, Abel T (2004) Behavioral impairments caused by injections of the protein synthesis inhibitor anisomycin after contextual retrieval reverse with time. Proc Natl Acad Sci USA 101:4667-4672.

LeDoux JE (2000) Emotion circuits in the brain. Annu Rev Neurosci 23:155-184.

Maren S (2001) Neurobiology of Pavlovian fear conditioning. Annu Rev Neurosci 24:897-931.

Maren S, Ferrario CR, Corcoran KA, Desmond TJ, Frey KA (2003) Protein synthesis in the amygdala, but not the auditory thalamus, is required for consolidation of Pavlovian fear conditioning in rats. Eur J Neurosci 18:3080-3088.

McGaugh JL (1966) Time-dependent processes in memory storage. Science 153:1351-1358.

Miller R, Springer A (1974) Implications of recovery from experimental amnesia. Psychol Rev 81:470-473.

Miller RR, Kraus JN (1977) Somatic and autonomic indexes of recovery from electroconvulsive shock-induced amnesia in rats. J Comp Physiol Psychol 91:434-442.

Myers KM, Davis M (2002) Systems-level reconsolidation: reengagement of the hippocampus with memory reactivation. Neuron 36:340-343.

Nader K (2003) Memory traces unbound. Trends Neurosci 26:65-72.

Nader K, Schafe GE, LeDoux JE (2000a) The labile nature of consolidation theory. Nat Rev Neurosci 1:216-219.

Nader K, Schafe GE, Le Doux JE (2000b) Fear memories require protein synthesis in the amygdala for reconsolidation after retrieval. Nature 406:722-726.

Pavlov IP (1927) Conditioned reflexes. New York: Dover.

Paxinos G, Watson C (1986) The rat brain in stereotaxic coordinates. Sydney: Academic.

Pedreira ME, Maldonado H (2003) Protein synthesis subserves reconsolidation or extinction depending on reminder duration. Neuron 38:863-869.

Quartermain D, McEwen BS (1970) Temporal characteristics of amnesia induced by protein synthesis inhibitor: determination by shock level. Nature 228:677-678.

Salinska E, Bourne RC, Rose SP (2004) Reminder effects: the molecular cascade following a reminder in young chicks does not recapitulate that following training on a passive avoidance task. Eur J Neurosci 19:3042-3047.

Sara SJ (2000) Retrieval and reconsolidation: toward a neurobiology of remembering. Learn Mem 7:73-84.

Schafe GE, LeDoux JE (2000) Memory consolidation of auditory pavlovian fear conditioning requires protein synthesis and protein kinase $\mathrm{A}$ in the amygdala. J Neurosci 20:RC96(1-5).

Serota RG (1971) Acetoxycycloheximide and transient amnesia in the rat. Proc Natl Acad Sci USA 68:1249-1250.

Suzuki A, Josselyn SA, Frankland PW, Masushige S, Silva AJ, Kida S (2004) Memory reconsolidation and extinction have distinct temporal and biochemical signatures. J Neurosci 24:4787-4795.

Vianna MR, Szapiro G, McGaugh JL, Medina JH, Izquierdo I (2001) Retrieval of memory for fear-motivated training initiates extinction requiring protein synthesis in the rat hippocampus. Proc Natl Acad Sci USA 98:12251-12254.

Walker MP, Brakefield T, Hobson JA, Stickgold R (2003) Dissociable stages of human memory consolidation and reconsolidation. Nature 425:616-620.

Young AG, Galluscio EH (1971) Recovery from ECS-produced amnesia. Psychon Sci 22:149-151. 